\title{
A PRÁTICA DO APRENDIZADO ORGANIZACIONAL
}

\section{THE ORGANIZATIONAL LEARNING PRACTICE}

\author{
Leonardo Ensslin'; Marcelo Luís de Campos \\ 'Universidade Federal de Santa Catarina - UFSC - Florianópolis - Brasil \\ ensslin@deps.ufsc.br \\ ${ }^{2}$ Universidade Federal de Santa Catarina - UFSC - Florianópolis - Brasil \\ marcelo_campos@deps.ufsc.br
}

\begin{abstract}
Resumo
Através de uma análise comparativa entre as Organizações que Aprendem (SENGE, 1990), o modelo genérico de Criação do Conhecimento Organizacional (NONAKA et al, 1997) e a metodologia de Multicritério de Apoio à Decisão - MCDA (ROY, 1993) e (ENSSLIN et al, 2001), pretende-se mostrar que a metodologia MCDA, fundamentada no paradigma construtivista, é potencialmente um meio para viabilizar as organizações de aprendizagem ou mesmo para a criação do conhecimento organizacional. Através da aplicação desta metodologia, pode-se obter o foco e a convergência dos conceitos relacionados nas teorias de aprendizagem organizacional citadas.
\end{abstract}

Palavras-chave: Apoio à Decisão, Aprendizado Organizacional, Gestão do Conhecimento.

\section{Introdução}

A era atual é caracterizada pela competição acirrada entre as organizações, na qual a única certeza tida é a mudança. O momento é indescritivelmente incomparável, extremamente dinâmico e muito instável, exigindo das organizações uma forte capacidade de adaptação às mudanças. Sendo assim, possuir um "bom desempenho" já não garante a sua sustentação no mercado no qual está inserida, é, pois necessário um "desempenho de referência” viabilizado através de recurso humanos mais bem capacitados, dos melhores produtos ou serviços e da melhor rentabilidade do negócio.

Inseridos também neste contexto, estão os executivos que necessitam tomar decisões cada vez mais alinhadas com os propósitos da organização. Estes profissionais devem possuir uma altíssima capacidade de adaptação, habilidades analíticas privilegiadas e os devidos meios para aplicá-las, um instinto para oportunidades aguçado, sagacidade para negócios, velocidade e precisão na tomada de decisão e, principalmente, foco no que realmente é fator de incremento da 
performance organizacional. É preciso encontrar meios de distinguir o que realmente é importante daquilo que é menos importante, identificar as variáveis nas quais deve-se focar e as variáveis nas quais deve-se prestar menos atenção - "manter o foco atualizado" em todas as atividades desenvolvidas.

A aprendizagem organizacional e a Gestão do Conhecimento, temas que vem se tornando clássicos da teoria das organizações, respondem com afinco ao cenário exposto, principalmente, por vincularem-se ao conceito de inovação. Cada vez mais, para criar e estabilizar posições de desempenho competitivo, algumas organizações estão dedicando-se ao desenvolvimento e aplicação de conceitos como conhecimento e aprendizagem. $\mathrm{O}$ desenvolvimento do conhecimento organizacional representa um recurso intangível capaz de conferir-lhes vantagem competitiva e um importante ativo estratégico a fim de que garantam posição de destaque no meio em que atuam.

A motivação deste artigo decorre do desenvolvimento de uma experiência ("de um case") com uma metodologia que tem se mostrado muito competitiva para ajudar em situações como as que foram apresentadas anteriormente. A metodologia em questão, além de incorporar as vantagens dos conhecimentos dos especialistas da área, os integra em uma forma sistêmica e sinergética conferindo-lhes um potencial competitivo até então não disponível.

\section{A Criação do Conhecimento Organizacional}

Dentre os autores que discorrem sobre o tema, destacam-se Nonaka e Takeuchi (1997) que propõem uma epistemologia para a criação do conhecimento organizacional, partindo da distinção entre o conhecimento tácito e o conhecimento explícito. Por criação de conhecimento organizacional entende-se "a capacidade que uma empresa tem de criar conhecimento, disseminá-lo na organização e incorporá-lo aos produtos, serviços e sistemas (NONAKA et al, 1997)". Nonaka et al (1997) explicam ainda que a criação do conhecimento organizacional é um processo espiralado, que surge quando a interação entre o conhecimento tácito e o conhecimento explícito eleva-se dinamicamente de um nível ontológico inferior até os níveis mais altos.

O processo de criação do conhecimento (ver Figura 1) começa com o compartilhamento do conhecimento tácito, que corresponde à socialização, pois o conhecimento rico e inexplorado que habita os indivíduos precisa ser amplificado dentro da organização. Na seqüência, o conhecimento tácito compartilhado é convertido em conhecimento explícito na forma de um novo conceito, ou seja, trata-se do processo de externalização. Na terceira fase, o conceito criado precisa ser justificado, quando se determina se é relevante ou não a sua implementação. Já na quarta fase, sendo os conceitos validados, os mesmos são convertidos em arquétipos. A última fase amplia o conhecimento criado através da sua difusão interna na organização (NONAKA ET AL, 1997). 


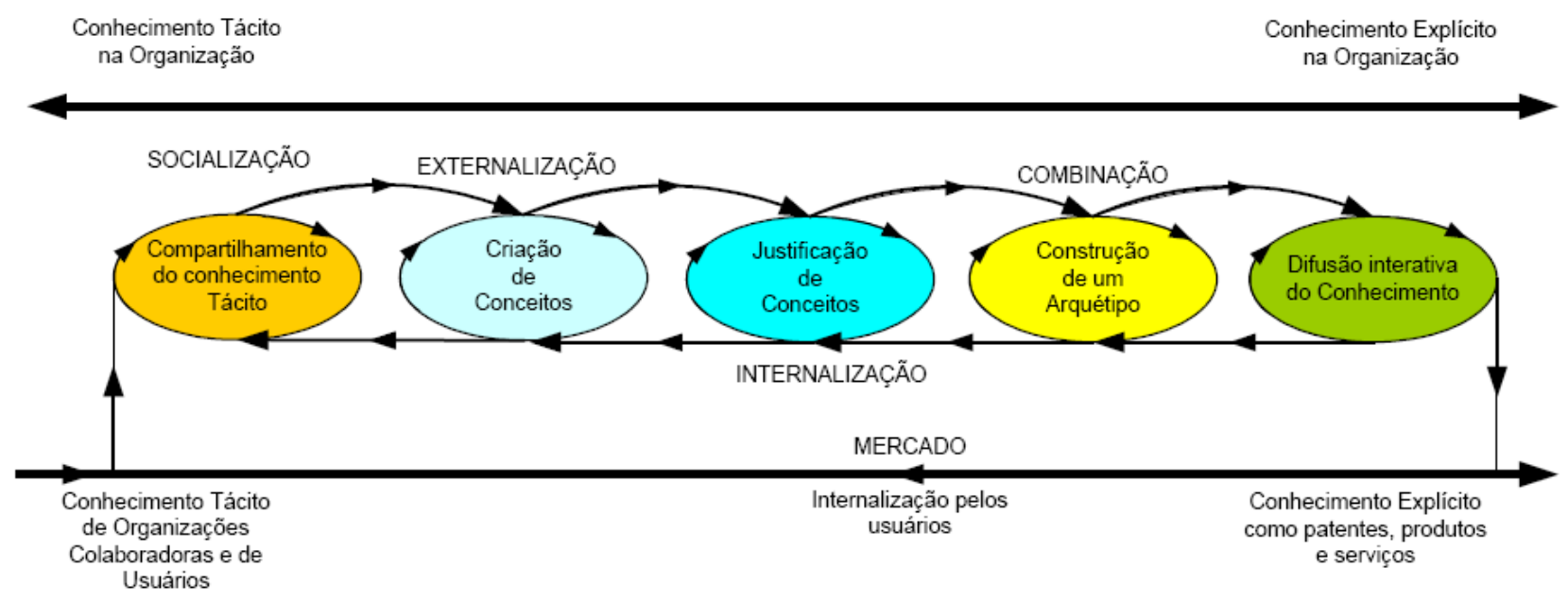

MODELO DE CINCO FASES DO PROCESSO DE CRIAÇÃO DO CONHECIMENTO

Nonaka et al (1997) entendem a criação do conhecimento organizacional como um processo que amplifica o conhecimento individual em nível organizacional e o cristaliza como parte da rede de conhecimentos da organização. Este conhecimento individual é de difícil formalização, pois se estabelece basicamente em uma dimensão cognitiva complexa, formada de modelos mentais, paradigmas, imagens e percepções individuais, e de uma visão e entendimento particular da realidade dos indivíduos que compõem a organização.

\section{Organizações de aprendizagem}

De acordo com Ensslin et al (1997), uma das melhores respostas ao cenário descrito na introdução deste artigo são as “organizações que aprendem” de Senge (1990). É eminente a contribuição de Senge (1990), que destaca as cinco disciplinas, incorporadas pelo pensamento sistêmico como os meios para a obtenção do aprendizado organizacional. Sumariamente, apresentam-se a seguir as idéias desenvolvidas pelo autor supra citado.

De acordo com Senge (1990), cada uma das cinco disciplinas apresenta uma dimensão vital no desenvolvimento de uma organização que aprende, são elas: domínio pessoal, modelos mentais, a construção de uma visão compartilhada, aprendizado em equipe e o pensamento sistêmico.

O domínio pessoal, por sua vez, é a disciplina de continuamente esclarecer e aprofundar a nossa visão pessoal e ver a realidade objetivamente. As pessoas com alto nível de domínio pessoal conseguem concretizar os resultados mais importantes para elas, e são capazes de separar o que realmente é relevante em suas vidas, ou seja, seus objetivos pessoais, e ainda, aprendem a visualizar com clareza a realidade do momento. Nesta linha, contrapor o alvo, ou seja, o objetivo desejado com a situação atual, é o que gera a tensão criativa propulsora da solução dos problemas. 
Os modelos mentais são orientadores da forma de pensar e agir das pessoas, são "paradigmas" profundamente fixados. De acordo com Senge (1990), muitas vezes não estamos conscientes de nossos modelos mentais e dos impactos que estes causam sobre a nossa forma de pensar e agir. Muitas idéias não seguem adiante nas organizações devido aos modelos mentais profundamente enraizados nas mentes dos atores responsáveis pela implementação das mesmas, desta forma, toda a atenção deve ser dispensada a esta disciplina, pois se atribuí também a ela o fato de, por vezes, existirem as resistências ocultas às mudanças organizacionais que ameaçam o status quo.

A construção de uma visão compartilhada é o que propicia o foco e a energia para o aprendizado, criando um compromisso ao invés do simples envolvimento ou aceitação. A declaração de missão das organizações representa esta visão compartilhada por todos de forma explícita. No entanto, é importante que esta declaração seja mais do que simplesmente um pedaço de papel outorgado pelo líder principal da organização, sendo necessária a existência de formas de viabilização prática desta visão.

O aprendizado organizacional, ou o aprendizado em equipe enfrenta o paradoxo do "mais significa menos", ou seja, muitas vezes, individualmente encontramos soluções adequadas para problemas complexos e coletivamente, ou em grupo, não conseguimos chegar nem sequer a soluções consideradas de modesto desempenho. Senge (1990) exemplifica bem com o seguinte questionamento: "Como uma equipe formada por gerentes com QI acima de 120, pode ter, coletivamente, um QI de 63?’. Sabe-se que tal questionamento não está mesmo fora do contexto da realidade de algumas organizações.

Esta disciplina começa com o diálogo, ao melhor dizendo, com a capacidade dos indivíduos de abnegarem-se de suas idéias preconcebidas. O aprendizado em equipe é crucial, Senge (1990) cita que "as equipes, e não os indivíduos, são a unidade de aprendizagem fundamental nas organizações modernas".

O pensamento sistêmico é a quinta disciplina, busca a integração entre as outras quatro já citadas. É a estrutura conceitual que tem por objetivo tornar a visão do conjunto clara. O pensamento sistêmico catalisa a integração entre as outras disciplinas, lembrando que "a soma das partes pode exceder o todo". Um exemplo comum da ausência da prática da quinta disciplina - e também não raramente encontrado - pode ser a definição e a formalização das missões organizacionais sem uma compreensão das forças propulsoras para o alcance das mesmas, o que põe em causa a credibilidade das intenções de alguns líderes.

\section{Aprendizagem organizacional}

Garvin (1993) define a organização que aprende como "uma organização capacitada para criar, adquirir e transferir conhecimentos e modificar seu comportamento de modo a refletir novos 
conhecimentos e insights". Sendo assim, propõe cinco eixos para a aprendizagem organizacional:

- Resolução sistemática de problemas: este primeiro eixo privilegia a utilização de métodos científicos para diagnosticar problemas ao invés do tradicional "feeling". Ratifica a utilização de dados ao invés de suposições para a tomada de decisão e o uso generoso de ferramentas estatísticas para a organização dos dados e estruturação dos problemas;

- Experimentação: consiste da procura e experimentação sistemáticas de novos conhecimentos, aonde a utilização de método cientifico é essencial. Sendo que a experimentação deve ser concomitante à resolução sistemática de problemas;

- Aprendizagem com a experiência passada: está baseada na revisão sistemática das experiências passadas, com ênfase na avaliação dos sucessos e fracassos a fim de planejar as ações no presente e futuro;

- Aprendizagem com o ambiente externo: a utilização de experiências vivenciadas por outras organizações também é um dos meios de se promover a aprendizagem. Significa o ganho de uma nova perspectiva através da análise sistemática das experiências vividas por outras organizações. A prática de benchmarking é potencialmente utilizada para a análise das melhores performances, a avaliação das mesmas e também a implementação, se for o caso;

- Difusão do conhecimento: decorre da transferência do conhecimento através de toda a organização como meio de capitalização do mesmo. Pode ser promovido através de vários processos como, por exemplo, educação e treinamento, padronização, entre outras técnicas de difusão e formalização do conhecimento.

Garvin (1993) cita que o melhoramento contínuo requer um compromisso com a aprendizagem, e que uma organização não pode progredir sem antes aprender algo novo. $\mathrm{O}$ autor enfatiza que os esforços que devem ser investidos a fim de promover o aprendizado organizacional são no sentido de evoluir do melhoramento contínuo para um compromisso com a aprendizagem através dos 3M's (do inglês meaning, management e measuring): o significado, o gerenciamento e a medição da aprendizagem. Sendo assim, é necessário primeiramente que se tenha uma definição de aprendizado organizacional que possibilite imprimir ações para que o mesmo seja operacionalizado.

De forma conclusiva, Garvin (1993) afirma ainda que as teorias sobre organizações de aprendizagem apresentadas, apesar de serem reconhecidamente de extrema relevância e de incontestável valia, não proporcionam um direcionamento mais prático. Garvin justifica que as teorias apresentadas restringem sua atuação sobre a percepção abstrata. Em outras palavras, as teorias apresentadas, segundo a sua visão, não demonstram um referencial para a ação, ou seja, não apontam para a operacionalização prática da aprendizagem organizacional. O grande desafio em questão é o de se estabelecerem os "mecanismos" ou as "ferramentas" para que, de forma prática e 
focada, se possa "operacionalizar" as organizações de aprendizagem, ou ainda, proporcionar a criação do conhecimento organizacional.

\section{A perspectiva proposta pela metodologia MCDA acerca do aprendizado organizacional}

Em pouquíssimas atividades as pessoas têm tanta "vivência" e "experiência" quanto na tomada de decisões. Tomamos dezenas, talvez centenas de decisões todos os dias. No entanto, em ambientes organizacionais, raramente as decisões são tomadas por indivíduos únicos, mesmo que exista, ao final, um responsável único pelo resultado a ser apresentado (ENSSLIN ET AL, 2001).

Roy (1996) avalia que as decisões são tomadas quando escolhemos ou não fazer alguma coisa. Sendo que, a decisão acontece em um processo ao longo do tempo, apesar de ser a tomada de decisão em si, um ato pontual. De fato, as decisões se dão em um processo caótico, no qual são confrontados os valores e as percepções individuais dos atores envolvidos com o problema.

Estas confrontações são trazidas à tona através do processo de interação entre os atores envolvidos, fazendo emergir os aspectos subjetivos destas mesmas percepções. O desenvolver dessa atividade de "emergir" estas "confrontações e interações" entre os atores é o que aqui chamamos de processo decisório (ROY, 1996). Entende-se que esta etapa pode se transformar em uma oportunidade para desenvolver, estruturar, disseminar e compartilhar os conhecimentos estratégicos, tácitos e operacionais dos atores a respeito do assunto que se deseja e, através de ações corretivas (decisões), aperfeiçoar o desempenho organizacional.

De acordo com Ensslin et al (2001), o processo de Apoio à Decisão pode ser dividido em três grandes fases:

\section{- A fase de estruturação do problema;}

- A fase de avaliação das ações potenciais;

- A fase de recomendações.

O autor ressalta ainda a característica de "recursividade" das mesmas, ou seja, o fato de se poder avançar e recuar com o progresso do desenvolvimento do conhecimento. Segundo Ensslin et al (2001), "Esta recursividade é fruto da própria geração de conhecimento aos participantes sobre o seu problema". De forma esquemática, está representado na figura 2 abaixo, o arquétipo proposto, ilustrando as etapas que decorrem no desenvolvimento da metodologia de apoio à decisão, baseada no paradigma construtivista, e a sua relação com o modelo de criação do conhecimento organizacional de Nonaka et al (1997). 
Figura 2 - Arquétipo proposto do processo de criação e estruturação do conhecimento organizacional (Nonaka et al, 1997) operacionalizado através da metodologia multicritério de apoio à decisão (Ensslin et al, 2001).

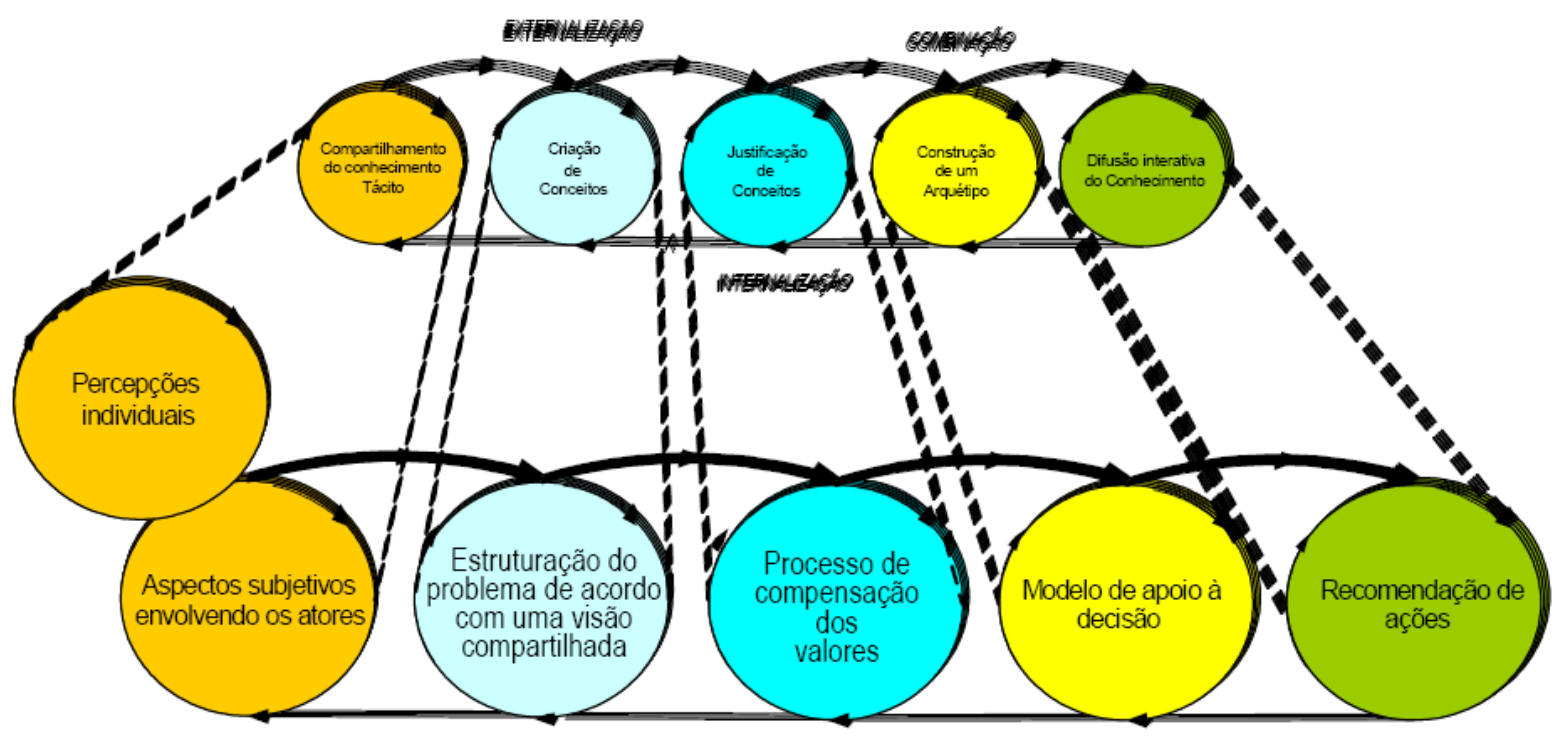

O processo de estruturação do conhecimento é essencial para se gerar um entendimento comum sobre o problema, orientar o processo de criação das ações e, por fim, servir de base para o processo de avaliação das mesmas. É também nesta fase do processo decisório que serão levados em conta os valores dos decisores a respeito da situação do problema, desta forma, procura-se estruturar o problema de acordo com uma visão compartilhada dos envolvidos no processo.

O objetivo dos modelos de apoio à decisão, fundamentados em uma visão construtivista, é de gerar conhecimento aos atores do processo decisório. Os modelos construídos através do processo de apóio à decisão devem permitir identificar oportunidades de aperfeiçoamento, bem como, devem servir também como base para que os atores entendam o impacto que as ações exercem sobre os seus valores. Dentro da visão da metodologia MCDA, os atores devem perceber que o seu trabalho é melhorar o desempenho do contexto e que as possíveis soluções são apenas os meios para tal, mas o foco é no aperfeiçoamento do desempenho, representado por seus objetivos (ENSSLIN ET AL, 2000).

Segundo a visão de Roy (1996), um modelo de apoio à decisão é apenas um fragmento da realidade, e deve ser compreendido e considerado como uma "ferramenta" cujo principal objetivo é entender e comunicar sobre esta realidade. Como o propósito do MCDA construtivista é de focar, organizar e expandir o conhecimento do contexto, e fazê-lo através da identificação e alinhamento dos objetivos do contexto com os da organização, a mesma pode ser considerada como um processo para desenvolver, estruturar e disseminar os conhecimentos em forma alinhada com os interesses da organização em questão.

A metodologia MCDA tem ainda uma última etapa - denominada de "Recomendações", onde é desenvolvido, de forma mais intensa, o processo de geração de ações para o contexto, a 
análise e também a avaliação de suas performances em cada aspecto julgado relevante. Neste momento, o modelo multicritério construído pode ser o meio para canalizar as discussões entre os envolvidos a respeito daquilo que é mais importante acerca do contexto decisório. Desta forma, dispõe-se aqui de um recurso para operacionalizar a difusão interativa do conhecimento sobre a situação.

Estando a metodologia MCDA-C fundamentada no paradigma do construtivismo, este pressupõe a noção de construção do conhecimento a partir da participação dos atores no processo decisório. Na figura 3 está demonstrado o processo estruturado de prática da aprendizagem organizacional, onde, aplicando-se a metodologia MCDA-C através da interação mediada pelo facilitador, são levados em conta tanto o sistema de valores quanto as percepções individuais dos atores envolvidos no processo de estruturação do conhecimento.

Neste caso, o conhecimento é estruturado através da modelagem de um dado contexto decisional, gerando no mesmo momento a aprendizagem necessária ao processo de recomendação de ações potencializadoras da melhoria do desempenho organizacional. Através da mesma estrutura aqui proposta, é possível a disseminação do conhecimento destinado a promover e sustentar o desempenho global da organização. Esse processo se viabiliza por meio do desenvolvimento do aprendizado no respectivo domínio de cada comunidade de prática, da estruturação do conhecimento e da partilha do mesmo entre estes grupos existentes na organização, ou seja, a metodologia MCDA-C pode ser compreendida como sendo a própria prática do conhecimento.

Conforme Wenger (2004), entende-se por comunidades de prática um grupo de indivíduos reunidos em torno de uma determinada área de conhecimento comum, o domínio como sendo a própria área de conhecimento em torno da qual a comunidade está inserida, e a prática são as ferramentas e métodos desenvolvidos pelas comunidades e partilhados por ela. 


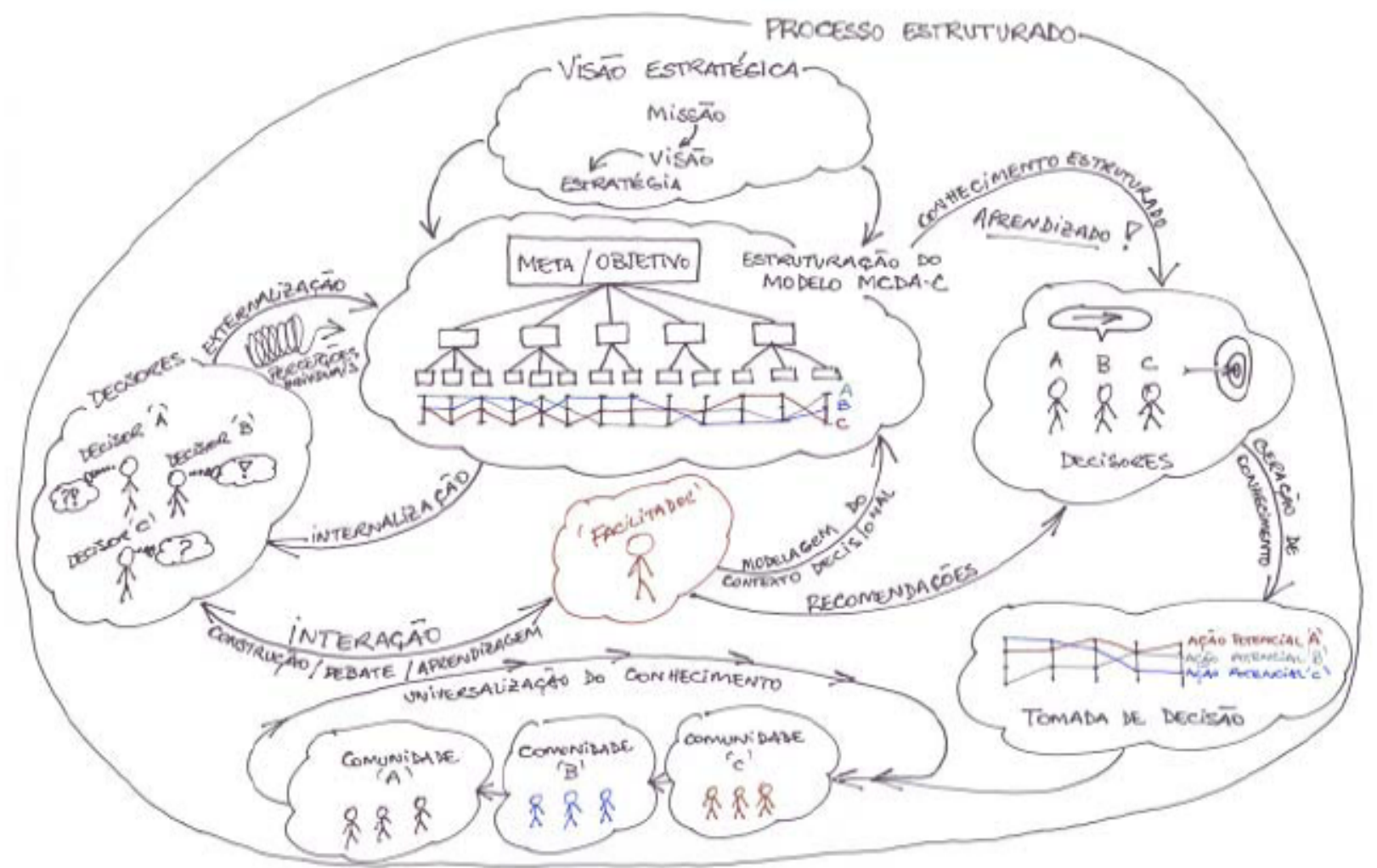

\section{Conclusão}

A partir dos argumentos de Senge (1990), quando da análise das cinco disciplinas: domínio pessoal, modelos mentais, visão compartilhada, aprendizado organizacional e pensamento sistêmico, Nonaka et al (1997) concluíram que os mesmos "não apresentam nenhuma idéia sobre as formas nas quais o conhecimento pode ser criado".

Garvin (1993), na mesma linha de pensamento, avalia que, apesar da relevância da teoria, as idéias apresentadas por Senge (1990) não apresentam um referencial claro para a ação a fim de promover a aprendizagem e a criação do conhecimento organizacional. Através dos cinco eixos possíveis para a aprendizagem organizacional, Garvin (1993) estende a sua contribuição ilustrando cada um dos eixos com algumas práticas de idéias passíveis de implementação.

Dadas as limitações apresentadas, este artigo mostra que, através do arquétipo proposto, a metodologia multicritério de apóio a decisão, fundamentada no paradigma construtivista de Roy (1993) e Ensslin et al (2001), quando aplicada para estruturação, avaliação e recomendação de ações em problemas complexos, pode promover resultados satisfatórios para a criação e gerenciamento do conhecimento organizacional. O presente artigo evidencia ainda que existe estreita correlação entre as três abordagens, que são constatadas na medida em que seus conceitos são justapostos. 
No entanto, as questões acerca do tema de aprendizagem ou da criação do conhecimento organizacional estão ainda bem distantes do esgotamento. O propósito aqui é de, cada vez mais, buscar os meios de se obter o aprendizado, estruturar e disseminar o conhecimento e permitir uma aceleração do processo de evolução das organizações.

\begin{abstract}
Through a comparative analyses between the Learning Organizations (SENGE, 1990), the Organizational Knowledge Creation Model (NONAKA et al, 1997) and the Multicriterial Decision Aided - MCDA (ROY, 1993) and (ENSSLIN et al, 2001), the intention is to present the MCDA, based on the constructivist paradigm, as a path to get the learning organizations or to create the organizational knowledge. MCDA constructivist could give the way to converge concepts disposed on the organizational learning theories.
\end{abstract}

Key-words: decision aid, organizational learning, knowledge management.

\title{
Referências
}

ENSSLIN, L. - Avaliação e Recomendações da Metodologia Multicritério de Apoio à Decisão: disciplina MCDA IIIPPGEP-EPS-CTC-UFSC. Notas de Aula. Slides, 2003.

ENSSlin, L.; MONTIBELlER NETO, G.; M. NORONHA, S.M. - Apoio á Decisão. Florianópolis/SC: Insular, 296p., 2001 .

ENSSLIN, L.; DUTRA. ,A.; ENSSLIN, S.R. - MCDA: A Constructivist Approach to the management o Human Resources at a Governmental Agency, Intl. Trans. In Op. Res-ITORS - Published by Elsevier, v. 7, p. 79-100, 2000.

ENSSLIN, L.; ZANELLA, I.J.; ENSSLIN, E.R. - Decision Aiding as a Basis to a Learning System Implementation. In: XVII ENEGEP - ENCONTRO NACIONAL DE ENGENHARIA DE PRODUÇÃO E 3O. CONGRESSO INTERNACIONAL DE ENGENHARIA INDUSTRIAL, Gramado/RS. Anais do XVII ENEGEP - Encontro Nacional de Engenharia de Produção e 3o. Congresso Internacional de Engenharia Industrial. Gramado/RS. CDROM (t7405), 1997.

GARVIN, D. - Building a Learning Organization. Harvard Business Review. Boston, p. 78-91, jul./ago, 1993.

NONAKA, I.; TAKEUCHI, H. - Criação do Conhecimento na Empresa. Campus, Rio de Janeiro/RJ, 358 p, 1997.

ROY, B. - Multicriteria Methodology for Decision Aiding, Dordrecht: Kluwer Academic Publischer, 1996.

ROY, B. - Decision Science or Decision Aid Science, European Journal of Operational Research - EJOR, v. 66, n. 2-3, p. 184-203, abr, 1993.

SENGE, P. M. - A Quinta Disciplina. São Paulo: Best Seller, 1990.

Von KROGH, G.; ICHIJO, K.; NONAKA, I. - Facilitando a criação do conhecimento: reinventando a empresa com o poder de inovação. Rio de Janeiro: Campus, 2001.

WENGER, E. - Knowledge management as a doughnut: Shaping your knowledge strategy through communities of practice. Ivey Business Journal, London, Jan/Feb, 2004. 
Dados completos do Segundo autor

Marcelo Luís de Campos

Laboratório de Multicritério em Apoio à Decisão LabMCDA

Universidade Federal de Santa Catarina - UFSC

Campus Universitário - Trindade - Caixa Postal 476

CEP: 88040-900 - Florianópolis - Santa Catarina

Telefone: (48) 331-7022 - FAX (48) 331-7075

E-mail: marcelo_campos@deps.ufsc.br

Recebido para publicação em: 22/12/2005

Aceito para publicação em: 20/02/2006 